\title{
Incidence of surgical infections in patients undergoing craniotomy during the period 2007-2015
}

\author{
Vilà E, Fernández-Candil J.L., Moltó L, Rodríguez C, Pacreu S, Sadurní M.
} Anaesthesiology Dpt, Parc de salut Mar, Barcelona, Spain

\section{Introduction:}

The surgical infection incidence in patients undergoing craniotomy is around 5\%. In our hospital the infection rate was much higher (14\% in 2007 and $12.5 \%$ in 2008) for what was being studied and a multidisciplinary working group was created.

\section{Objective:}

Determine the nosocomial infection incidence in scheduled craniotomies during the period 2007-2015 and assess the response after the implementation of various measures to reduce infection.

\section{Method:}

All patients underwent elective craniotomy were followed up to 30 days after surgery. Several measures were implemented: 1) Epidemiological study of infections, 2) Culture surgical instruments at the sterilization room, 3) Limit the number of people in the operating room (OR), the door openings and avoid surgical staff shift changes, 4) Preparation of a pre, intra and postoperative checklist, 5) Expansion of OR for storage of boxes of material, 6) Change disinfected skin wash and shaving head, 7) Exclusive OR for neurosurgery. The implementation of some of the measures was performed simultaneously; however there are some important dates: 1) $2010^{\text {th }}$, creation of the checklist and multidisciplinary working group, 2) From 2012 to the present, microbiological analysis of all surgical infection in neurosurgery, 3) $2014^{\text {th }}$ change of antibiotic prophylaxis

\section{Results:}

Overall surgeries \& Annual infection rate 2007-2015

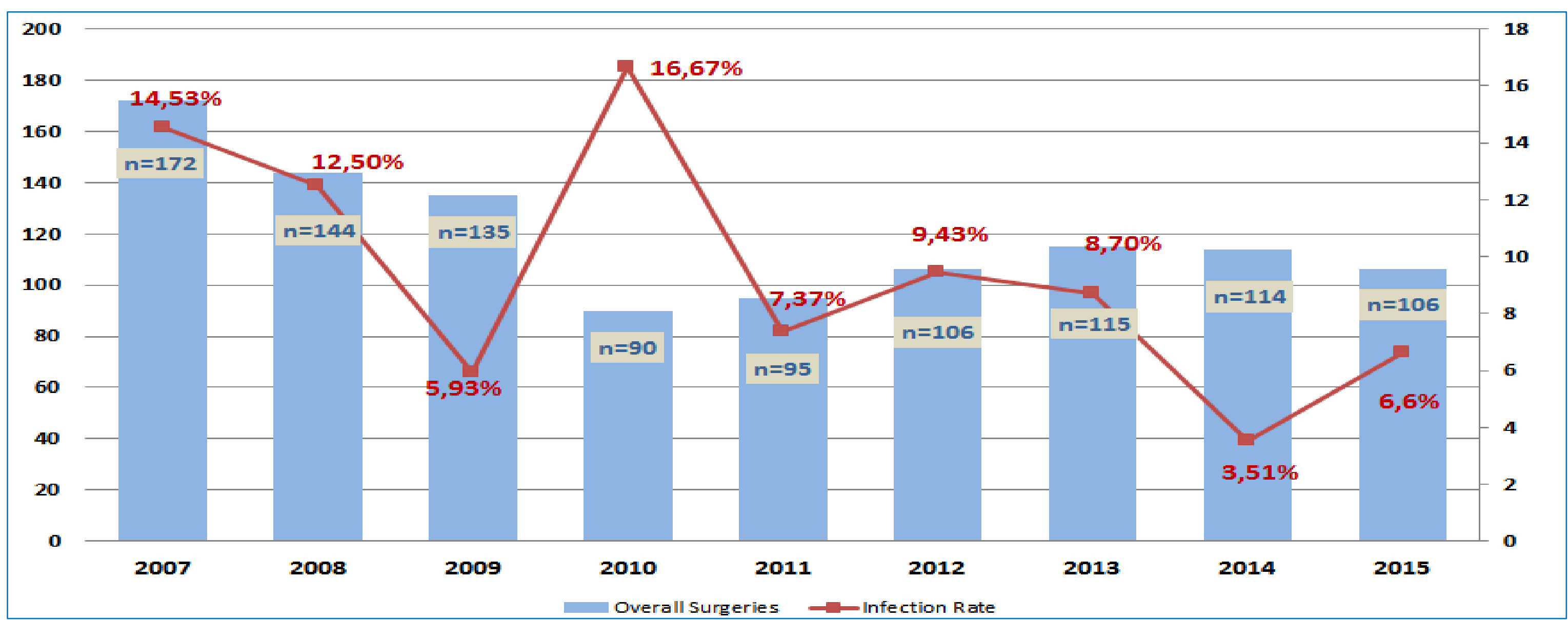

Microbiological analysis of postoperative wound infections made during the period 2012-2013 evidenced 20 postoperative infections of 221 procedures. $53 \%$ of the isolated pathogens were gram-negative bacteria (GNB) that were not covered with the empirical antibiotic prophylaxis with cefazolin and in 2014 ${ }^{\text {th }}$ was replaced with cefuroxime. From 2014-2015 ${ }^{\text {th }}$ eleven of the 220 craniotomies were infected and 4 cocoas gram-positive and 2 GNB were identified.

\section{Conclusions:}

Knowing the local epidemiology of infections is critical to guide both prophylaxis and empirical therapy. The multidisciplinary approach of the surgical wound infections helps enhance the results. 